\title{
Ein IgA-Antiphospholipid-Syndrom - eine seltene klinische Variante
}

\author{
S. Lutze; M. Ahmed, W. Konschake, G. Daeschlein, M. Jünger \\ Klinik- und Poliklinik für Haut-und Geschlechtskrankheiten, Universitätsmedizin Greifswald, Greifswald
}

\begin{abstract}
Schlüsselwörter
Antiphospholipid-Syndrom, beta-2-Glycopro-

tein-IgA, Vaskulopathie, Ulcus cruris

Zusammenfassung

Das Antiphospholipid-Syndrom vom IgA-Subtyp ist eine Variante innerhalb der Erkrankungsgruppe des Antiphospholipid-Syndroms. Es ist ebenso mit einem deutlich erhöhten Risiko für thrombembolische Komplikationen assoziiert wie das Antiphospholipid-Syndrom vom IgG, IgM-Isotyp. Das klinische Bild an der Haut ist mannigfaltig, es reicht von einer Livedo racemosa, über Ulcera cruris bis hin zu disseminiert auftretenden, vaskulitisch imponierenden Läsionen. Vor allem die vaskulitischen Veränderungen lassen sich am ehesten durch die Eigenschaften des Immunglobulin-Subtyps erklären. Zur weiteren Diagnosesicherung und Abgrenzung anderer Differentialdiagnosen ist die Hinzunahme von IgA-Antikörpern sowie eine Hautbiopsie oft hilfreich.
\end{abstract}

Korrespondenzadresse

Dr. med. Stine Lutze

Klinik- und Poliklinik für Haut-und Geschlechtskrank-

heiten, Universitätsmedizin Greifswald

Tel. + 493834866770 ,

E-Mail: stine.lutze@uni-greifswald.de

\section{Hintergrund}

Das Antiphospholipid-Syndrom (APS), erstmalig im Jahre 1959 durch. P. Hughes und P. G. I. Stovin beschrieben (1), ist eine systemisch verlaufende Autoimmunerkrankung, charakterisiert durch das gehäufte Auftreten von thromboembolischen Ereignissen (2). Gemäß aktueller Studienlage wird eine Häufigkeit des Antiphospholipid-Syndroms von 1-5\% in der Allge-

\section{Keywords}

Antiphospholipid syndrome, beta-2-glycoprotein IgA, vasculopathy, ulcus cruris

\section{Summary}

IgA subtype antiphospholipid syndrome is a variant within the antiphospholipid syndrome group. It is also associated with a significantly increased risk of thrombembolic complications, as is the Ig antiphospholipid syndrome, IgM isotype. The clinical picture on the skin is varied, ranging from livedo racemosa to ulcers cruris and disseminated vasculitic lesions. Especially the vasculitic changes can best be explained by the properties of the immunoglobulin subtype. The addition of IgA antibodies and a skin biopsy is often helpful for further diagnosis and differentiation of other differential diagnoses.

IgA antiphospholipid syndrome - a rare clinical variant

Phlebologie 2018; 47: 261-264

https://doi.org/10.12687/phleb2437-5-2018

Eingegangen: 09. Juli 2018

Angenommen: 18. Juli 2018

English version available at:

www.thieme.de/phlebo
Im Jahre 1999 wurden erstmalig Kriterien für die Einstufung der Erkrankung durch ein Expertenkomitee in Sapporo, Japan festgelegt (6). Gemäß dem Consensuspapier vom 2006 (7) gelten nun folgende Kriterien für die Krankheitseinstufung:

Klinische Kriterien:

a) Vaskuläre Thrombosen - eine oder mehr Episoden von arteriellen, venösen oder Kleingefäß-Thrombosen

b) Schwangerschaftsmorbiditäten-

- Eine oder mehr Totgeburten morphologisch normaler Feten

- Eine oder mehr Frühgeburten

- Drei oder mehr aufeinanderfolgende Spontanaborte

Laborchemische Kriterien:

a) Anticardiolipin Antikörper vom IgG und/oder IgM Isotyp im Serum oder Plasma im mittleren oder hohem Titer an zwei oder mehr Untersuchungszeitpunkten mit 12 wöchigem Abstand mittels standardisiertem Verfahren gemessen

b) Beta-2-Glycoprotein I Antikörper vom IgG und/oder IgM Isotyp im Serum oder Plasma im mittleren oder hohem Titer an zwei oder mehr Untersuchungszeitpunkten mit 12 wöchigem Abstand mittels standardisiertem Verfahren gemessen

c) Lupus-Antikoagulanzien im Plasma an zwei oder mehr Untersuchungszeitpunkten, in 12 wöchigen Abständen gemessen (gemäß guidelines of the International Society on Thrombosis and Hemostasis)

\section{Kasus}

\section{Krankheitsanamnese}

Krankheit tritt häufig in Schüben verlaufend auf, wobei die Intervalle von Wochen, Monaten bis Jahren reichen können $(4,5)$. 

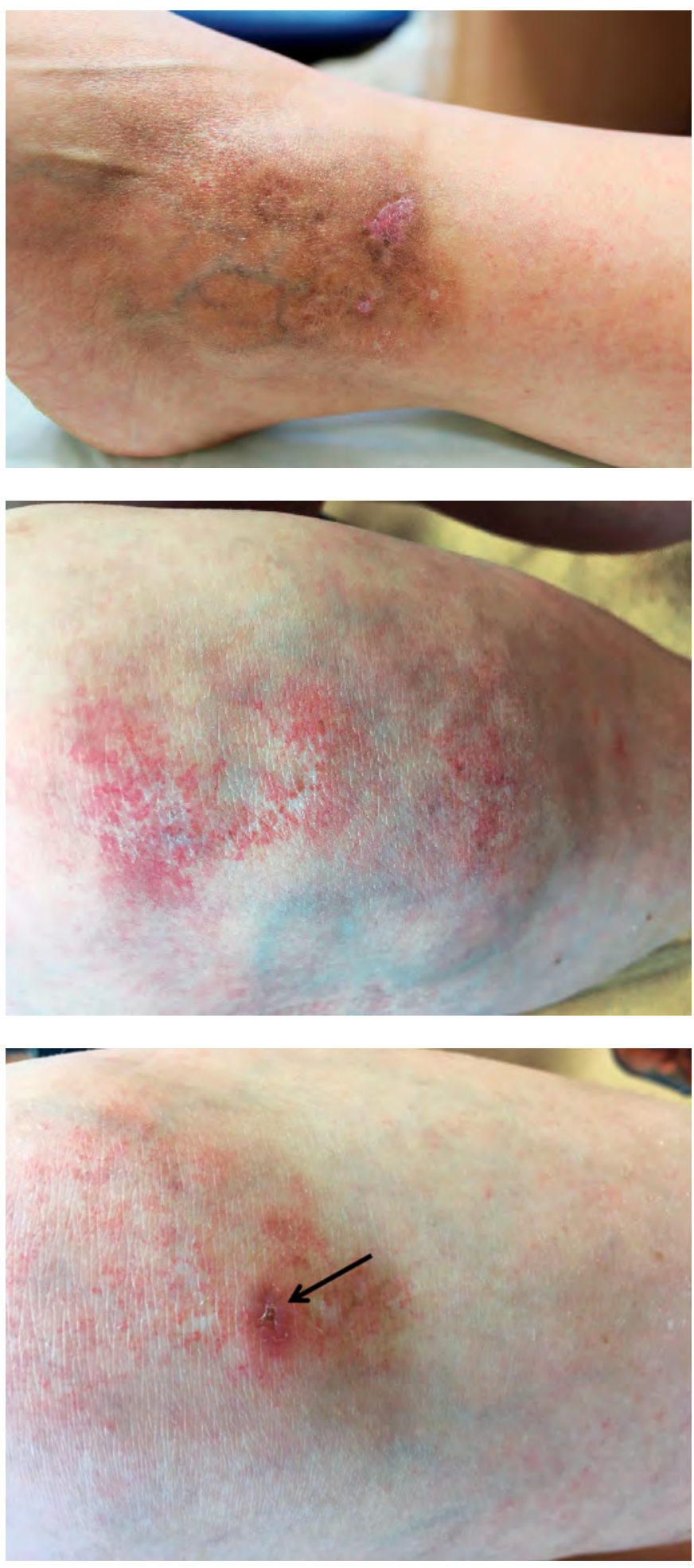

Abb. 1

In Abheilung befindliche Ulzerationen oberhalb des linken Malleolus lateralis neben Hyperpigmentierungen und Atrophie blanche ähnlichen Arealen

Abb. 2

Disseminiert an beiden Beinen bestehende, gruppiert angeordnete teleangiektatische Veränderungen

Abb. 3

Disseminiert an beiden Beinen bestehende, gruppiert angeordnete teleangiektatische Veränderungen neben porzellan-farbenen kleinen Narben und einer floriden Ulzeration (Pfeil)

bereich im Jahre 2016 in unserer Ambulanz vor. Eine weiterführende Ursachenabklärung wurde veranlasst. Die VaskulitisDiagnostik inklusive gängiger Antiphospholipid-Antikörper wie Antikörper gegen Cardiolipin, beta-2-Glycoprotein I sowie

fach in der Vergangenheit unauffällig, sodass trotz fehlender duplexsonographischer und klinischer Korrelation unter Diagnose einer chronisch venösen Insuffi- zienz, eine Kompressionstherapie extern empfohlen wurde.

Die sorgfältige Anamneseerhebung ergab weder Frühaborte noch Fehlgeburten in der Krankheitsgeschichte. Arterielle oder venöse thrombotische Ereignisse der großen Gefäße waren ebenfalls nicht zu eruieren. Eine Befundverschlechterung betont in den Sommermonaten wurde von der Patientin beschrieben.

\section{Klinische Manifestation}

Bei Vorstellung sahen wir wie ausgestanzt imponierende, $0,5 \mathrm{~cm}$ messende Ulzerationen an den Knien lateral sowie im Bereich des Malleous medialis et lateralis beidseits mit umgebender Hyperpigmentierung der Dermis. Neben diesen Veränderungen zeigten sich an beiden Beinen Felder mit gruppiert stehenden ektasierten kleinen Gefäßen ( Abb. 1-3). Typische Zeichen einer chronisch venösen Insuffizienz wie ein Unterschenkelödem, eine Corona phlebectatica paraplantaris sowie tropischen Hautveränderungen an den Unterschenkeln waren bei Vorstellung nicht zu sehen. In der Dermatoskopie zeigen sich auffällige Gefäßmuster in Form von korkenzieherartig konfigurierten Gefäßen und Mikrothromben in den ektasierten Gefäßen neben kleinen Ulzerationen ( $\$$ Abb. 4-6).

\section{Laborchemische Diagnostik}

Bei klinisch weiter bestehendem Verdacht auf eine vaskuläre Genese der Veränderungen erfolgte zum einen eine Biopsie an der Haut und bei dermatoskopisch dringendem Verdacht auf eine Vaskulopathie die Erweiterung der serologischen Untersuchungen auf ergänzende Antikörper-Profile. Hier zeigte sich dann ein hochtitriger Wert für den Beta-2-Glycoprotein-IgA Subtyp.

\section{Histologisches Bild}

Das histologische Bild zeigt den Befund einer Vaskulopathie im Bereich der kleinen Gefäße und schließt eine Vaskulitis ursächlich aus. Unter einer unauffällig aufgebau- 
ten Epidermis zeigen sich gruppiert stehend vermehrt kleine dickwandige Gefäße (postkapilläre Venolen), welche Teilthrombosierungen aufweisen, daneben bestehen Erythrozytenextravasate ( $>$ Abb. 7). Ein perivaskuläres Infiltrat, wie es klassisch bei Vaskulitiden auftritt, kommt nicht zur Darstellung.

\section{Therapie und weiterer Krankheitsverlauf}

Die Therapie wurde leitliniengereicht mit Acetylsalicylsäure $100 \mathrm{mg}$ täglich, als thrombozytenaggregationshemmende

Therapie eingeleitet. Hierunter zeigte sich eine vollkommene Abheilung der anfänglich bestehenden Ulzerationen. Die Verlaufskontrollen bis ein Jahr nach Therapiebeginn zeigten eine Befundstabilität. Im Juli 2017 stellte sich die Patientin mit einem erneuten Krankheitsschub vor. Klinisch zeigten sich erneut disseminiert kleine Ulzerationen im Bereich des Malleolus medialis. Die laborchemische Untersuchung zeigte erneut einen hohen Titer Anstieg von Beta-2-Glycoprotein - IgA sodass wir parallel zu dem bereits laufenden Thrombozytenaggregationshemmer eine Systemtherapie mit Chloroquinphosphat 250mg/ Tag einleiteten. Bei weiterer Befundzunahme wurde im März 2018 die Systemtherapie mit Acetylsalicylsäure 100mg auf 300mg täglich erhöht. Hierunter stabilisierte sich der Befund, es traten keine neuen Ulzerationen auf, sodass zuletzt die Systemtherapie mit Chloroquinphosphat auf eine Tagesdosis vom 50mg pro Tag und Acetylsalicysäure auf $100 \mathrm{mg}$ reduziert werden konnte.

\section{Diskussion}

Seit einigen Jahren besteht in der klinischen Forschung Uneinigkeit über die Wertigkeit von den IgA-Antikörpern beim Antiphospholipid-Syndrom. Gemäß dem Consensus-Papier des 14. internationalen Antiphospholipid-Syndrom Kongresses gilt die Empfehlung bei starkem klinischem Verdacht auf das Vorliegen eines Antiphospholipid-Syndroms bei jedoch negativen Titern für IgM und IgG Antiphospholipid-

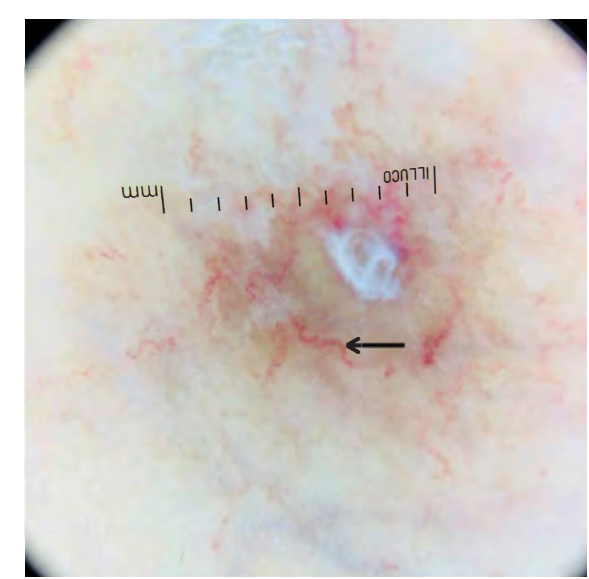

Abb. 4 Dermatoskopie: es kommt ein kleines UIcus zur Darstellung mit aufgelagertem CremeRest umgeben von korkenzieherartig konfigurierten Gefäßen (Pfeil)

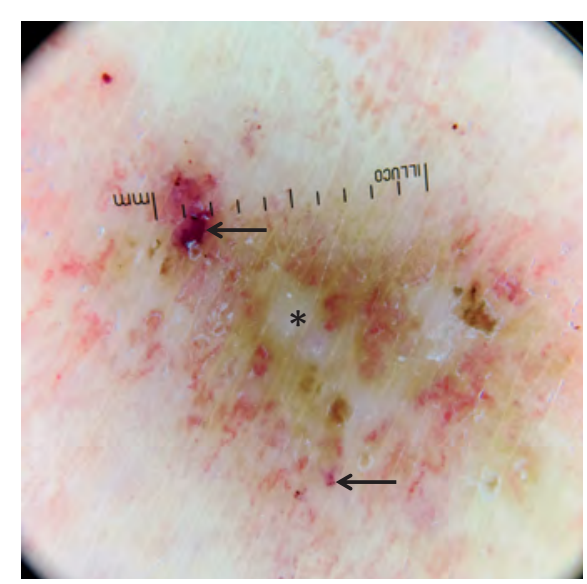

Abb. 5 Dermatoskopie: es zeigen sich abgeheilte porzellan-farbene Areale $\left({ }^{*}\right)$, umgeben von Pigmentablagerungen und ektasierten, z.T. frisch thrombosierten Gefäßen (Pfeil)

Abb. 6

Dermatoskopie: es zeigen sich abgeheilte porzellan-farbene Areale $\left({ }^{*}\right)$, umgeben von ektasierten, korkenzieherartig konfigurierten Gefäßen und einem kleinen aktiven Ulcus (Pfeil)

Tab. 1 Erweiterung der serologischen Untersuchungen auf ergänzende Antikörper-Profile: Hier zeigte sich ein hochtitriger Wert für den Beta-2-Glycoprotein-IgA Subtyp.

\begin{tabular}{l|l}
\hline Antikörper & Titer \\
\hline Cardiolipin-lgM & $<2 \mathrm{Mpl} / \mathrm{ml}($ Normbereich <2Mpl/ml) \\
\hline Cardiolipin-IgG & $2,1 \mathrm{Gpl} / \mathrm{ml}($ Normbereich $<12 \mathrm{Gpl} / \mathrm{ml})$ \\
\hline Beta-2-Glycoprotein - IgM & $5 \mathrm{RE} / \mathrm{ml}($ Normbereich $<20)$ \\
\hline Beta-2-Glycoprotein - IgG & $<2 \mathrm{RE} / \mathrm{ml}$ (Normbereich <20) \\
\hline Beta-2-Glycoprotein - IgA & $>\mathbf{2 0 0 ~ R E} / \mathrm{ml}$ (Normbereich <20) \\
\hline
\end{tabular}

Antikörper den IgA-Subtyp zu bestimmen (8).

In unserem Kasus sind die typischen präpartalen Komplikationen nicht festzustellen. Dies könnte ursächlich darin begründet liegen dass im Gegensatz zum IgG, IgA nicht plazentagängig ist. Nichts desto trotz lässt der klinische Aspekt der disseminiert auftretenden kleinsten Ulzerationen in der Haut neben vorbestehenden atypischen Gefäßmustern in Form von Teleangiektasien, nicht viele Differentialdiagnosen zu. Neben der Gruppe der hochinflammatorischen Small Vessel Vasculitiden steht die Vaskulopathie als eine Erkrankung aus dem Bereich der Koagulopathien. Histologisch können diese rasch voneinander abgegrenzt werden. Das Antiphospho- 


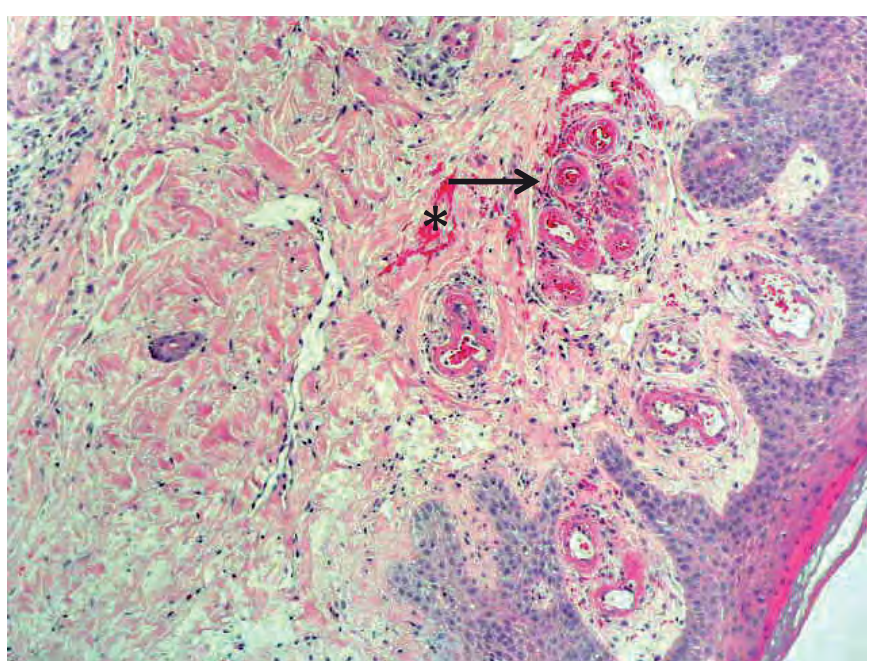
Musters) mit hyalinen

lipid-Syndrom, zu den Vaskulopathien zugehörig, führt in den Gefäßen der Dermis zu Mikrothromben, durch die hierdurch bedingte Minderperfusion kommt es zum Gewebsuntergang des dazugehörigen Hautabschnitts. Im Gegensatz hierzu führt die Vaskulitis über eine inflammatorische Veränderung der Gefäßwände zu einer Extravasation von Erythrozyten durch die erhöhte Gefäßpermeabilität zu den typischen palpablen Purpura. Sekundär kommt es durch Entzündung der Gefäße zum Verschluss der Lumina und hierdurch bedingt zur Hautnekrose.

Ähnlich wie die z.T. auch nur auf die Haut begrenzte IgA-positive Vaskulitis (9) limitiert sich bei unserer Patientin die IgA Vaskulopathie ebenfalls auf das Hautorgan. Daher lassen sich gewisse Parallelen zwischen diesen beiden IgA-Immunkomplex vermittelten Krankheitsbildern ziehen.

Im Fall der IgA-positiven leukozytoklastischen Vaskulitis (Purpura SchoenleinHennoch) führt die reduzierte Aktivität von beta1,3-Galaktosyltransferase in den peripheren B-Zellen zu einer abnormalen Glykosylierung von IgA-1. Das pathologische IgA führt zu einer reduzierten Immunabwehr an den Schleimhäuten. Dies
Abb. 7

HE Färbung, 10x. Histologisches Bild einer Vaskulopathie: subepidermal zeigen sich vermehrt kleine Gefäße, gruppiert angeordnet (Pfeil) (entspricht dem klinischen Korrelat des korkenzieherartigen Wandverdickungen ohne perivaskuläres Infiltrat, daneben bestehen Erythrozytenextravasate $\left({ }^{*}\right)$

\section{Literatur}

1. Hughes JP, Stovin PGI. Segmental pulmonary artery aneurysms with peripheral venous thrombosis. $\mathrm{Br}$ J Dis Chest 1959; 53: 19-27. doi:10.1016/S0007-0971(59)80106-6

2. Cervera R. Antiphospholipid sydrome. In: Thrombosis Research 2017; 151: 43-S47. DOI: 10.2016/S0049-3848(17)30066-X.

3. Gómez-Puerta JA, Cervera R. Diagnosis and classification of the antiphospholipid syndrome. In: Journal of autoimmunity 2014; 48-49: 20-25. DOI:10.1016/j.jaut.2014.01.006.

4. Cervera R, Khamashta MA, Shoenfeld Y, Camps MT, Jacobsen S, Kiss E et al. Morbidity and mortality in the antiphospholipid syndrome during a 5-year period: a multicentre prospective study of 1000 patients. Ann Rheum Dis 2009; 68: 1428-1432.

5. Cervera R, Serrano R, Pons-Estel GJ, CeberioHualde L, Shoenfeld Y, de Ramón E et al. Morbidity and mortality in the antiphospholipid syndrome during a 10-year period: a multicentre prospective study of 1000 patients. Ann Rheum Dis 2015; 74: 1011-1018.

6. Wilson WA, Gharavi AE, Koike T, Lockshin MD, Branch DW, Piette JC et al. International consensus statement on preliminary classification criteria for definite antiphospholipid syndrome: report of an international workshop. Arthritis Rheum 1999; 42: 1309-1311.

\section{Zusammenfassung}

Bei ursächlich unklaren, rezidivierend und disseminiert auftretende Ulzerationen vor allem an den unteren Extremitäten und primär negativer autoimmunologischer Serolgie sollte an das seltene Erkrankungsbild eines IgA-Antiphospholipid-Syndroms gedacht werden. Hier ist neben der entsprechend erweiterten Labordiagnostik, eine Biopsie oft hilfreich.

\section{Interessenkonflikt}

Nach Angaben der Autoren bestehen keine Interessenkonflikte.

\section{Ethische Richtlinien}

Für das Manuskript wurden keine Studien an Menschen oder Tieren durchgeführt.
7. Miyakis S, Lockshin MD, Atsumi T, Branch DW, Brey RL, Cervera R et al. International consensus statement on an update of the classification criteria for definite antiphospholipid syndrome (APS). J Thromb Haemost 2006; 4: 295-306.

8. 14th International Congress on Antiphospholipid Antibodies Task Force Report on Antiphospholipid Syndrome Treatment Trends Doruk Erkan a, Cassyanne L. Aguiar a, Danieli Andrade b, Hannah Cohen c,d, Maria J. Cuadrado e, Adriana Danowski f, Roger A. Levy g, Thomas L. Ortel h, Anisur Rahman c,d, Jane E. Salmon a, Maria G. Tektonidou i, Rohan Willis j, Michael D. Lockshin a

9. Sunderkötter C, Pappelbaum K, Ehrchen J. Hautarzt 2015; $66: \quad 589 . \quad$ https://doi. org/10.1007/s00105-015-3661-6

10. Davin J-C. Henoch-Schonlein purpura nephritis. Pathophysiology, treatment, and future strategy. Clinical journal of the American Society of Nephrology, CJASN 2011; 6 (3): 679-689. DOI: 10.2215/CJN.06710810. 